\title{
OPERATIONAL RISK - SCENARIO ANALYSIS
}

\author{
Milan Rippel, Petr Teplý *
}

\begin{abstract}
:
This paper focuses on operational risk measurement techniques and on economic capital estimation methods. A data sample of operational losses provided by an anonymous Central European bank is analyzed using several approaches. Multiple statistical concepts such as the Loss Distribution Approach and the Extreme Value Theory, including scenario analysis method, are considered. Custom plausible loss events defined in a particular scenario are merged with the original data sample and their impact on capital estimates and on the financial institution as a whole is evaluated. Two main questions are assessed - what is the most appropriate statistical method to measure and model operational loss data distribution and what is the impact of hypothetical plausible events on the financial institution. The g\&h distribution was evaluated to be the most suitable one for operational risk modeling. The method based on the combination of historical loss events modeling and scenario analysis provides reasonable capital estimates and allows for the measurement of the impact of very extreme events on banking operations.
\end{abstract}

Keywords: operational risk, scenario analysis, economic capital, loss distribution approach, extreme value theory, stress testing.

JEL Classification: G21, G32, C15

\section{Introduction}

There are some widely known operational risk events of severe magnitude that occurred in the last few years; the most publicly known examples of operational risk include a loss of $\$ 7.3$ billion at Société Générale in 2007 or more recently the $\$ 65$ billion Ponzi scheme by Mr. Bernard Madoff and the $\$ 8$ billion bank fraud of Sir Allen Stanford. Operational risk events also occurred during the pending global crisis, such as failed risk management processes or mortgage frauds committed by applicants when cheating on their income in order to secure a loan (Teplý, 2010).

* Institute of Economic Studies, Faculty of Social Sciences, Charles University In Prague, Opletalova 26, CZ - 110 00, Praha 1 (milanrippel@seznam.cz; teply@fsv.cuni.cz). The findings, interpretations and conclusions expressed in this paper are entirely those of the authors and do not represent the views of any of the authors' institutions. Financial support for this research from The Grant Agency of Charles University (GAUK 31610/2010 - Optimal Methods of Operational Risk Management); The Czech Science Foundation, project The Institutional Responses to Financial Market Failures, under No. GA P403/10/1235; The IES Institutional Research Framework 2005-2010 under No. MSM0021620841; and The Czech Science Foundation, project The Implications of The Global Crisis on Economic Capital Management of Financial Institutions under No. GA 403/10/P278 is gratefully acknowledged. 
Additionally, the New Basel Capital Accord (Basel II), valid since 2007, newly introduced a capital requirement for operational risk (in addition to credit and market risk). This fact has further fostered the focus on operational risk management.

In this paper we focus on modelling and stress testing the economic and regulatory capital set aside to cover unexpected operational risk losses of an anonymous Central European bank (BANK). There are two main questions this paper is focused on:

1. What is the appropriate statistical method to model operational risk loss data distribution and measure reasonable capital estimates for the institution?

2. What is the impact of extreme events defined in particular extreme case scenarios on the capital estimates and on the financial institution?

Several statistical distributions are used to model loss severity distribution and compute capital estimates. It is expected that the best results will be provided by a distribution that can reasonably model the body as well as the heavy right tail of the data sample. On the other hand, techniques that focus just on the tail of the distribution might not provide consistent results if the tail is contaminated by additional extreme loss events defined by scenarios. The distribution that is expected to be the most suitable for modelling the operational risk data is the g\&h distribution used by Dutta, Perry (2007). So the test hypotheses can be stated as:

$\mathrm{H}_{1}$ : The $\mathrm{g} \& \mathrm{~h}$ distribution provides consistent capital estimates for scenario analysis method;

$\mathrm{H}_{2}$ : Extreme Value Theory (EVT) provides consistent capital estimates for scenario analysis method.

Once these hypotheses are assessed, the effects of unanticipated extreme events on the financial institution can be evaluated. It is assumed that the bank would not be able to cover the worst case joint scenario losses, because the loss amounts would exceed bank capital reserves. On the other hand, the bank should be able to cover average joint scenario losses.

The first rigorous studies on operational risk management were introduced in late 1990s, through published studies by Prof. Embrechts. Given the scarcity and confidentiality of operational risk loss data, there are only a few papers that explore the specifics of operational risk data and are able to measure operational risk exposure with the accuracy and precision comparable with other sources of risk. The most comprehensive studies are de Fontnouvelle, Jordan, Rosengren (2005), Degen, Embrechts, Lambrigger (2007), Embrechts, Frey, McNeil (2005), Mignolla, Ugoccioni (2006), Chernobai, Rachev, Fabozzi (2007) and Dutta, Perry (2007). A scenario analysis method, the method used in this paper, is discussed in papers from Cihak (2004), Arai (2006), Kuhn, Neu (2004) or Rosengren (2006). More recently, Chalupka, Teplý (2008), Rippel, Teplý (2010) and Teplý (2010) provide a detailed overview of operational risk management methods.

This paper is organized as follows: Section 2 provides an overview of operational risk concepts related to Basel II requirements. Section 3 provides an overview of the methodology used. Section 4 analyzes the data sample of BANK and proposes distributions that can best model the data sample. Section 5 provides a theoretical overview of stress testing and scenario analysis methodology. In Section 6 the loss 
events defined in particular scenarios are merged with the original data sample and new capital estimates are computed. Finally, Section 7 provides a conclusion and proposes areas for future research.

\section{Operational Risk Background and Basel II requirements}

\subsection{Basic terms}

The most common definition of operational risk is given in Basel II as "the risk of loss resulting from inadequate or failed internal processes, people and systems or from external events. This definition includes legal risk, but excludes strategic and reputational risk." (BCBS, 2006) The operational risk encompasses those risks, not covered under credit and market risk that have a measurable financial impact.

For operational risk modelling, it is crucial to distinguish between regulatory and economic capital. Regulatory capital is the amount of capital used for capital adequacy computation under Basel II. Economic capital is "a buffer against future, unexpected losses brought about by credit, market, and operational risks inherent in the business of lending money" (Mejstř́ik, Pečená and Teplý, 2008). Banks are expected to keep in reserve the necessary amount of economic capital to comply with Basel II Pillar II rules.

Regulatory capital covers unexpected losses only to a VaR confidence level $99.9 \%$ set by Pillar I of Basel II. For economic capital, banks typically set the VaR confidence level according to their operational risk exposure or use alternative measurement approaches - i.e. expected shortfall (Chernobai, 2007)

\subsection{Basel II operational risk measurement techniques}

Basel II sets three operational measurement methodologies for calculating operational risk capital charge in a continuum of increasing sophistication and risk sensitivity (BCBS, 2006). The first two approaches - Basic Indicator Approach (BIA) and Standardized Approach (SA) - are top-down approaches, because the capital charge is allocated according to a fixed proportion of a three-year average of the sum of net interest and net non-interest income (Basel Income Ratio). The third approach Advanced Measurement Approach (AMA) - is a bottom-up approach, because the capital charge is estimated based on actual internal operational risk loss data.

Under the AMA, the regulatory capital requirement equals the risk measure generated by the bank's internal operational risk measurement system using the quantitative and qualitative criteria that are given in Basel II. One of the AMA techniques is the Loss Distribution Approach (LDA) which uses statistical methods to measure the regulatory and economic capital a bank should allocate. LDA works with the database of past operational risk events. Another AMA technique is the Scenario Analysis (SCA) which is further described in Section 5. 


\section{Methodology}

\subsection{General remarks}

Empirical evidence proves that operational risk data have certain specifics, that causes techniques used for the assessment of credit and market risks unsuitable for operational risk management. From this point of view, operational risk management has more in common with insurance and actuarial mathematics. Consequently, insurance methodology can be successfully applied to operational risk assessment, for example when considering Extreme Value Theory (EVT).

The operational risk data are specific by the fact that there exist infrequent events that cause very severe losses to financial institutions. "Banks must be particularly attentive to these losses as these cause the greatest financial consequences to the institutions" (Chernobai, Rachev and Fabozzi, 2007).

On the other hand, the majority of the loss events are characterized by high frequency but low severity. Those events are relatively unimportant for a bank and can often be prevented using risk mitigation techniques or covered by provisions. When considering statistical distribution of operational risk loss severity data the "existing empirical evidence suggest that the general pattern of operational loss data is characterized by high kurtosis, severe right-skewness and a very heavy right tail created by several outlying events" (Chernobai, 2007). Distributions fitting such data are called leptokurtic. As will be shown later, the data sample provided by BANK exhibits the same characteristics.

\subsection{Models for operational risk measurement}

Two fundamentally different approaches are used to model operational risk:

- The top-down approach

- The bottom-up approach

The top-down approach quantifies operational risk without attempting to identify the events or causes of losses while the bottom-up approach quantifies operational risk on a micro-level being based on identified internal events. The top-down approach group includes, among others, the Risk Indicator models that rely on a number of operational risk exposure indicators to track operational risks and the Scenario Analysis and Stress Testing Models that are estimated based on the what-if scenarios.

The bottom-up approaches include actuarial type models that have two key components - frequency and loss severity distributions for modelling historical operational risk loss data sample. The capital charge is then computed as the value of $\mathrm{VaR}_{0.999}$ measure of the one-year aggregate distribution loss.

\subsection{Frequency distributions}

The studies based on empirical data suggest that choice of frequency distribution is not as important as an appropriate choice of loss severity distribution (de Fontnouvelle, de Jesus-Rueff, Jordan and Rosengren, 2003). The survey of studies done by Chernobai, 
Rachev and Fabozzi (2007) suggest that the Poisson distribution will be a reasonable solution for modelling operational risk frequency. Features of Poisson distribution are explained in Chalupka, Teplý (2008) or Rippel (2008).

\subsection{Loss severity distributions}

Several distributions were used to model loss severity. The distributions differ in the number of parameters they use. The list ranges from a simple one parameter exponential over the two parameter gamma, Weibull and lognormal distributions to four parameter g\&h distribution (see de Fontnouvelle, de Jesus-Rueff, Jordan and Rosengren, 2003 or Rippel, Teplý, 2008).

The $\mathrm{g} \& \mathrm{~h}$ distribution is the most advanced parametric distribution that will be used in this paper. It is "a strictly increasing transformation of the standard normal distribution $Z$ defined by:

$$
X_{g, h}(Z)=A+\frac{B}{g}\left(e^{g Z}-1\right) e^{\frac{1}{2} h Z^{2}}
$$

where $A, B, g$ and $h \geq 0$ are the four parameters of the distribution" (Dutta and Perry, 2008). The parameters are estimated using the following algorithm. $\hat{A}$ is equal to median of the data sample $X_{0,5}$. The $\hat{g}$ parameter is defined as a median of

$$
g_{p}=-\left(\frac{1}{Z_{p}}\right) \log \left(\frac{X_{1-p}-X_{0,5}}{X_{0,5}-X p}\right),
$$

where $X_{p}$ is the $p^{\text {th }}$ percentile of g-distribution and $Z_{p}$ is the $p^{\text {th }}$ of standard normal distribution. The other two parameters are determined using the OLS regression of $\log$ $(U H S)$ on $Z_{p}^{2} / 2$, where $U H S$ is an upper half spread defined as

$$
U H S=\frac{g\left(X_{1-p}-X_{0,5}\right)}{e^{-g Z p}-1} .
$$

The $\hat{B}$ is estimated as the exponentiated value of the intercept of this regression and the $\hat{h}$ is estimated as the coefficient of that regression.

\subsection{Extreme Value Theory}

Extreme Value Theory (EVT) is a branch of statistics that focuses on the extreme phenomena - the rare events that are situated in a tail of a particular probability distribution. There are several techniques for the EVT - each of them uses different method to pick up the low frequency/high severity loss events. They differ in how they set a threshold to cut loss data distribution into two parts - the body and the tail. Under the EVT, the body is being modeled using a different method (e.g. empirical sampling) and the tails are being modeled using specific EVT methods. There are two ways to select tail observations from a data sample - Block Maxima Method (BMM) and Peak Over Threshold Method (POTM). 


\subsubsection{Block maxima method}

The Block Maxima Method (BMM) divides data sample into independent blocks of the same size and considers the highest observation from such a block. This model would be useful, if the extreme events were equally distributed over the whole time interval. "For very large extreme loss observation $x$, the limiting distribution of such normalized maxima is the Generalized Extreme Value (GEV)" (Chernobai, Rachev and Fabozzi, 2007). The probability density distribution function of GEV distribution has a form of:

$$
f(x ; \mu, \sigma, \xi)=\frac{1}{\sigma}\left[1+\xi\left(\frac{x-\mu}{\sigma}\right)\right]^{-1 / \xi-1} e^{-\left[1+\xi\left(\frac{x-\mu}{\sigma}\right)\right]^{-1 / \xi}} \text { for } 1+\xi\left(\frac{x-\mu}{\sigma}\right)>0,
$$

where $x$ refers to block maxima observations, $\mu \in R$ is the location parameter, $\sigma>0$ is the scale parameter and $\xi$ is the shape parameter. The GEV distribution can be divided into three cases based on the value of the shape parameter (Chalupka and Teplý, 2008). The most important case called the Fréchet or the type II extreme value (EV) distribution is for $\xi>0$. The tail of the Fréchet distribution is slowly varying and thus suitable for modelling high severity operational risk data.

\subsubsection{Peak over threshold method}

The POTM uses all observations that exceed certain high threshold level. As argued by Embrechts, Frey, McNeil (2005), these models are more frequently used in practice for operational risk exposure measurement. The limiting distribution for the POTM is the generalized Pareto distribution (GPD) with the probability density function in the form of:

$$
f(x ; \xi, \mu, \sigma)=\frac{1}{\sigma}\left(1+\frac{\xi(x-\mu)}{\sigma}\right)^{\left(-\frac{1}{\xi}-1\right)},
$$

where $x$ refers to the data exceeding the threshold, $\mu \in R$ is the location parameter, $\sigma>0$ is the scale parameter and $\xi$ is the shape parameter.

Similarly to GEV, also the GPD has special cases based on the value of the shape parameter. The most important case from operational risk modelling point of view is when $\xi>0$. In this case the GPD has very heavy tails. The GPD parameters can be again estimated by using either the MLE or the PWM methods - for more details see Teplý, Chalupka (2008).

A critical task for designing the GPD distribution is to set an appropriate threshold level. This level should be set to be sufficiently high to fit extreme events. But on the other hand, the filtered data sample should not be limited too much in order to provide reasonable statistical evidence. Several approaches to solve this optimization task exist. The most commonly used one relies on the visual observation of the mean excess plot, which is defined as the mean of all differences between the values of the data exceeding threshold level $u$ and $u$. In case of the GPD the empirical mean excess function can be formalized into the following equation: 


$$
e_{n}(v)=\frac{\sum_{j=1}^{n}\left(x_{j}-v\right) I\left(v<x_{j}\right)}{\sum_{j=1}^{n} I\left(v<x_{j}\right)}=\frac{\beta}{1-\xi}+\frac{\xi}{1-\xi} u
$$

where $v$ is the value above threshold level $u$. Threshold values against mean excess values provide the mean excess plot. If the data supports a GPD model, then this plot should become increasingly linear for higher values of $v$. A general practice is then to choose such $u$ for which the mean excess plot is roughly linear. Several other approaches for choosing the threshold exist - the most simple one is just to define the right tail as five or ten percent of the observations with highest loss.

\subsection{Goodness of fit tests}

The fit of distributions chosen should be tested by a set of Goodness of Fit Tests (GOFT) in order to avoid model risk. As Chalupka and Teplý (2008) note, an underestimated VaR would jeopardize the long-term ability of a bank to maintain a sufficient amount of capital reserves to protect against catastrophic operational losses, while a severely overestimated VaR would limit the amount of funds available for investment. There are two ways how to assess the GOFT - either by using in-sample GOFTs or backtesting. Backtesting is the opposite approach to stress testing which questions validity of a chosen model.

GOFTs are divided into two classes - visual tests and formal tests. Visual GOFTs compare empirical and hypothesized distributions by plotting them to a chart and comparing their characteristics. The most commonly used visual test is QuantileQuantile (QQ) plot which plots empirical data sample quantiles against the quantiles of the distribution that is being tested for fit - for more details on the QQ plot see Dutta, Perry (2007) or Rippel (2008).

Formal GOFTs test whether the data sample follows a hypothesized distribution. Empirical distribution function-based tests directly compare the empirical distribution function with the fitted distribution function. The tests belonging to this group are the Kolmogorov-Smirnov test (KS) and the Anderson-Darling (AD) test. Both of them state the same hypothesis but use different test statistics - for more details see Chalupka, Teplý (2008) or Rippel (2008).

\subsection{Aggregate loss distribution and capital charge estimates}

Once the frequency and severity loss distributions are evaluated, an aggregated risk exposure of the bank should be estimated. Both types of distributions are to be aggregated to a single model which estimates the total loss over a one-year period. The measure used for the estimation of required capital charge is the Value-at-Risk (VaR). In the context of operational risk, VaR is the total one-year amount of capital that would be sufficient to cover all unexpected losses with a high level of confidence such as 99.9\% (Chernobai, Rachev and Fabozzi, 2007).

Due to the fact that the cumulative distribution function is not linear in $\mathrm{X}$ nor in $\mathrm{N}$, analytic expressions for the compound distribution function do not exist and thus the function must be evaluated numerically. The most common technique relies on 
numerical approximation of the compound distribution function using the Monte Carlo simulations of loss scenarios. The algorithm is as follows:

1. Simulate a large number of Poisson random variates and obtain a sequence $n_{1}, n_{2}, \ldots n_{M C}$ representing scenarios of the total number of loss events in a one-year period.

2. For each of such scenarios $n_{k}$ simulate $n_{k}$ number of loss amounts using a specified loss severity distribution.

3. For each of such scenarios $n_{k}$ sum the loss amounts obtained in the previous step in order to obtain cumulative one-year losses.

4. Sort the sequence obtained in the last step to obtain the desired aggregate loss distribution.

The number of simulated observations differs. We will use 50,000 simulations for the purposes of this paper.

\section{Empirical Data Sample Analysis}

The data sample provided by BANK consists of 657 loss events. The following assumptions about the data sample were made:

- Exchange rate and inflation impacts are not considered, nominal values in EUR are used.

- The data sample is truncated from below, but the threshold is set to a very low value, so we do not use corrections for left truncation bias.

- The impact of insurance is not considered.

- While the SA uses $15 \%$ of Basel Income Ratio as a regulatory capital charge it is expected that using the LDA approach the reasonable interval for capital charge is $5-15 \%$ but this range might be broader for some banks. For instance, small banks with lower income might report higher the AMA than the SA as a result of incorporating of extreme losses in the model through stress testing.

The statistics for the whole sample show a significant difference between the mean and the median and a very high standard deviation which signals a heavy right tail ${ }^{1}$. The same information is given by the skewness measure. The high value of the kurtosis measure signals that the high standard deviation is caused by infrequent extreme observations. These findings suggest that the data sample provided by the BANK exhibits the specific features of operational risk data.

Table 1

Data Sample Statistics - Whole Sample in EUR

\begin{tabular}{|c|c|c|c|c|}
\hline Mean & Median & Std. deviation & Skewness & Kurtosis \\
\hline 41,738 & 3,114 & 280,538 & 14 & 225 \\
\hline
\end{tabular}

Source: BANK data sample.

1 While 80\% losses are lower than EUR 20,000 and 95\% are lower than EUR100,000, there are 4 cases where the loss exceeds EUR 2,000,000. 
The procedure described in Section 3.7 was used to aggregate the loss frequency and the loss severity distributions. The Monte Carlo simulation method with 50,000 trials was used for the parameter estimation as well as for the aggregation function. The regulatory capital estimates are provided as a percentage relative to the BANK average Basel Income Ratio over the last three-year period as required by CNB (2007). The regulatory capital is being measured as the ratio of $\mathrm{VaR}_{0.999}$ / Basel Income Ratio. The fit of the distributions to the sample data is evaluated by using the QQ plot, the $\mathrm{KS}$ and the $\mathrm{AD}$ tests. If the test statistics are higher than the critical value, then the null hypothesis that the particular distribution is able to model the operational risk data sample cannot be rejected.

The distributions mentioned above were used for modelling of loss severity distribution - namely the Empirical Sampling Method, lognormal, Weibull, exponential, gamma and g\&h parametric distributions and also EVT approaches - BMM and its two ways to set block maxima (max per month and max per quarter) and POTM with three ways to cut the extreme observations ( $\max 5 \%$, max $10 \%$ and the threshold method). Details are provided in Rippel (2008) or Rippel, Teplý (2009).

Table 2

Comparison of the Regulatory and Economic Capital Estimates

\begin{tabular}{|l|c|}
\hline \multicolumn{1}{|c|}{ Distribution } & Regulatory Capital \\
\hline Empirical & $2.31 \%$ \\
\hline G\&H & $4.43 \%$ \\
\hline BMM - Month & $14.95 \%$ \\
\hline POT - 5\% & $9.32 \%$ \\
\hline
\end{tabular}

Source: Authors

The conclusion for the LDA approach on the institution level is that only the g\&h, the BMM - max quarter and the POTM - max 5\% methods seem to be suitable for modelling the operational risk data for Basel II purposes and thus these methods will be used for the stress testing purposes. The results of these three methods plus the ESM are provided in the following table.

While employing the very high significance levels for EVT methods, the regulatory capital is being overestimated. Because of the high sensitivity of the EVT methods, it can be concluded that the $g \& h$ method provides more reasonable estimates than any EVT method used.

\section{Stress Testing and Scenario Analysis}

Because of the fact that the LDA approach is a historical one - the capital charge is estimated based on historical loss events - alternative methods for the operational risk management were developed. One of those methods is the scenario analysis or, generally, the stress testing. This method is supposed to examine whether a financial institution would be able to undergo exceptional risk losses. The stress testing should be used as a complementary approach to the VaR based LDA approach in order to 
ensure that a bank would be able to cover the losses even if a bank faces more severe risk events. "Whenever the stress tests reveal some weakness, management must take steps to manage the identified risks. One solution could be to set aside enough capital to absorb potential large losses. Too often, however, this amount will be cripplingly large, reducing the return on capital" (Jorion, 2007).

Stress testing methods are not comparable with each other. Neither the applications of the same stress tests to different financial institutions are comparable with each other, because the results are always bound to the specific risk profile of a financial institution. Adopting bad assumptions or using irrelevant scenarios would lead to irrelevant losses. Since the stress tests often define events with a very low probability of occurrence, the results become difficult to interpret and it is not clear which actions should be taken by the management in order to mitigate the risks. Quite often the results of stress tests appear unacceptably large and they are just ignored and dismissed as irrelevant. However, it is valuable to evaluate stress test results at different point of times and say whether the exposures to operational risk have changed.

The scenarios can be divided into two groups based on the type of event they define. The first group uses historical events like the $9 / 11$ terrorist attacks or the unauthorized trading in Societé Generalé in 2007. The second group, more widely used in practice, uses hypothetical scenarios. The scenarios are based on plausible risk events that have not happened yet, but a non-zero probability of their occurrence exists. A scenario can also be based on an analysis of a new product a bank is going to implement.

A typical scenario consists of the description of a complex state of the world that would impose an extreme risk event on financial institution, including: probabilities and frequencies of occurrence of the particular state of the world, business activities impacted by the event, maximum internal and external loss amounts generated by occurrence of such event and possible mitigation techniques. Even though such a scenario claims to be realistic, it is not possible to include all possible risk factors and features. However, risk managers are trying to define the scenarios, so that they correspond to the reality as much as possible (Jorion, 2007).

BANK combines all main approaches for the operational risk management including the scenario analysis. The aim of using scenarios is, as explained above, to get an overview about low frequency events that might have severe impact on BANK. BANK was using eight complex scenarios, which satisfy all the qualitative measures. The details on scenario definitions are provided in Rippel (2008).

The losses generated by the eight scenarios were aggregated with the capital estimates based on the original data sample using the LDA method and the results are evaluated in the following section.

\section{Applied Scenario Analysis}

Two main approaches were used to aggregate losses generated by the scenarios with the database of historical events. The first one uses a set of the worst-case losses defined by a particular scenario and aggregates these losses to the historical loss data sample. The second approach calculates an average loss given by probability distribution of the loss amounts defined by a particular scenario and aggregates those average losses to the historical loss data sample. In both cases the statistical distributions mentioned 
above, the g\&h, the POT - max 5\% and the BMM - max quarter, were used for the severity distribution of the aggregated loss sample. The Poisson distribution was used for the loss frequency. Both distributions were then aggregated and the regulatory capital estimates were computed by using the VaR.

In case of the g\&h loss severity distribution, the aggregation method of losses generated by the scenarios with the historical data sample is straightforward, because the additional losses are simply added to the database. However, in the EVT approaches, where the body and the tail of the distribution are being modeled by using a different statistical distribution, the aggregation algorithm is more complicated, because all of the losses generated by the scenarios belong to the tail of the aggregated database distribution and thus it directly impacts the EVT methods.

\subsection{Scenario definitions}

There are two groups of scenarios - first group consists of 8 scenarios (denoted as ID 1-8) defined by BANK. The second group consists of 4 scenarios that were created for the purpose of this paper ("custom scenarios" thereafter).

Table 3

Historical Scenarios List (loss amounts in EUR ths)

\begin{tabular}{|c|l|c|}
\hline ID & \multicolumn{1}{|c|}{ Scenario name } & Estimated loss \\
\hline $\mathbf{9}$ & Unauthorized trading - Kerviel & $112,000^{2}$ \\
\hline $\mathbf{1 0}$ & Process management failure - software loss & 7,300 \\
\hline $\mathbf{1 1}$ & External fraud - theft & 21,180 \\
\hline
\end{tabular}

Note: Scenarios 1-8 were took from BANK. ${ }^{3}$

Source: Authors

The losses generated by the 8 scenarios defined by BANK were merged with the historical loss events using the method explained above. These scenarios include such events as an electricity blackout or a fictitious deal. The average loss amounts for all of the scenarios are comparable to the other tail losses from the original historical data sample, thus these eight losses just enrich the original tail of the data. On the other hand, the magnitudes of the worst-case losses are apparently higher than the magnitude of the highest historical loss and so the right tail of such merged sample is much heavier than in the case of the historical data sample. However, one has to consider the very low probability that the worst-case scenario happe.

The following sections list custom scenarios defined by the authors. Three different historical scenarios were defined - the first one is based on an unauthorized trading, the second one is based on an external fraud and the third one is based on process management failure. All of those scenarios are based on concrete historical events. The estimated losses are quite high and thus they will be treated as the worst-case

2 The loss amount was rescaled using the ratio of total assets of Société General / total assets of BANK at the time of the loss event occurrence.

3 The definitions of those scenarios are confidential. 
losses. The historical scenarios will not be used for tests based on average losses. The definitions of those historical scenarios are provided in Rippel (2008).

The hypothetical scenario of BANK employee strike that would hit all the regions is considered. This type of scenario was chosen because of the historical evidence of similar events exists. The frequency of the scenario assessment was estimated to $1 \mathrm{per}$ 40 years based on the following facts: according to the historical data there were several bank employee strikes in recent years - two of them in India, one in Canadian TD Trust bank, one in the Greece National Bank. The duration of the strike ranged from 1 day to 1 week. It is assumed that the frequency of strikes would be quite low in the region of Central Europe. Usually the duration of such strike is limited only to several hours. There are none recent examples of an employee strike in a Central European bank.

The other important feature of a strike is its extent - a strike can range from one branch to a national strike. For the purpose of this paper it was assumed that the employee from all regions would go on strike at the same time. Such a scenario has a very low probability, but if it occurred it would have significant negative impact on the bank. The severity of this scenario depends on two factors - the extent and the duration of the strike. The extent was set to the whole country. The duration is assumed to range from one-hour strike to five business-day strike and the probability for each class was estimated according to the assumptions stated above.

A strike was assumed to cause four types of losses - the direct loss of lost revenue from branches was estimated based on the list of BANK branches and their revenues per day. The second source of loss are the costs connected with expenses on substitute employees that would be hired in order to maintain the bank critical operations. These costs increase with the duration of the strike and were estimated as a certain percentage of the direct loss of revenue. The third and the most severe type of loss is the loss of clients that was estimated as a proportion of yearly revenue from branches. While a 1-hour strike is not considered to have impact on customer satisfaction, in case of a whole week strike up to $5 \%$ of customers might decide to move to competitors. The last type of the loss is the costs connected with commercial disputes. The losses were estimated based on interest costs from non-realized transactions and estimated amount of dispute penalties.

Table 4

Strike Duration Probability Distribution ${ }^{4}$

\begin{tabular}{|c|l|c|}
\hline Probability & Duration & Estimated loss (EUR) \\
\hline $70 \%$ & 1 hour & 138,515 \\
\hline $25 \%$ & 1 day & $3,750,446$ \\
\hline $4 \%$ & $2-4$ days & $9,056,450$ \\
\hline $1 \%$ & 5 days & $20,890,382$ \\
\hline
\end{tabular}

Source: Authors

After taking into account all the assumed loss sources, the total loss was computed. The loss amounts and the probability distribution are listed in Table 4 - the loss amount grows as the duration of the strike increases.

4 The estimated loss amounts are based on concrete data - for more details see Rippel (2008). 
Table 5

Custom Hypothetical Scenario Details (loss amounts in EUR ths)

\begin{tabular}{|c|c|c|c|}
\hline ID & Scenario name & Worst-case loss & Average loss \\
\hline $\mathbf{1 2}$ & Employee strike - whole state & 20,890 & 1,606 \\
\hline
\end{tabular}

Source: Authors

The worst-case scenario is a strike that lasts five days. Under this case the loss amount reaches EUR 20 million, which is app. 2\% of the Basel Income Ratio. Such strike is considered to cause significant harm to BANK - especially by the loss of 5\% customers. Such scenario would also have very negative impact on the brand image and the credibility of bank would be damaged resulting in counter-party risk. The average loss size is significantly lower though - EUR 1.6 million.

\subsection{Tests - Scenario combinations and loss aggregation estimates}

Scenarios were combined into several packages, denoted by test IDs. Both the worstcase and the average losses are considered. The tests differ by the number of scenarios they use - at first all scenarios defined by BANK as well as the custom scenarios are considered. Then the number of scenarios considered is gradually decreased. Separate tests are run for the custom scenarios and for more frequent BANK scenarios.

The tests results provided in Table 6 suggest that the EVT method is not an appropriate one to model the operational risk data, because the results provided by both EVT methods (the BMM - max quarter and the POTM 5\%) were very sensitive to the number of the tail observations and to the length of the tail. If there is such extreme observation as the one defined by scenario ID9, then the capital estimates given by the EVT method would be unreasonably high and in some cases reaching the amount of BANK total assets. On the other hand, if the less extreme average loss case events are added to the data sample, then the capital estimates provided by both EVT methods are unreasonably low. The application of the EVT methods to the empirical data provides overestimated results for the worst-case scenarios and underestimated results for the average loss scenarios. However, it might be expected that the results provided by the EVT method would improve, as the number of observations increases.

In total, six tests were run. The aim was to analyze whether BANK would be able to handle particular combinations of events defined in the combination of scenarios. The impact of such joint scenario was evaluated. Scenarios were denoted by the IDs. For the hypothetical scenarios (ID 1-8 and 12) two levels of loss were considered - the worstcase level and the average level. For historical scenarios (ID9-11) only the worst-case loss amount is defined. Three statistical approaches were used to model the merged data sample - the g\&h, the EVT - BMM max quarter and the EVT - POT 5\% methods. 
Table 6

Comparison of Regulatory Capital Estimates - Average/Worst Loss Scenarios

\begin{tabular}{|l|l|c|c|c|}
\hline \multicolumn{1}{|c|}{ Test } & Scenario IDs & $\begin{array}{c}\text { BMM - Max M } \\
\text { Avg/Worst case }\end{array}$ & $\begin{array}{c}\text { POTM - 5\% } \\
\text { Avg/Worst case }\end{array}$ & $\begin{array}{c}\text { G\&h } \\
\text { Avg/Worst case }\end{array}$ \\
\hline Original & n.a. & $14.95 \%$ & $9.32 \%$ & $4.43 \%$ \\
\hline Test I & ID1-12 & $4.1 \% / 245 \%$ & $4.3 \% / 207 \%$ & $11.7 \% / 91 \%$ \\
\hline Test II & ID1-8 & $4 \% / 136 \%$ & $5.2 \% / 129 \%$ & $10 \% / 35.7 \%$ \\
\hline Test III & ID3-5,7-8 & $4.6 \% / 148 \%$ & $6.6 \% / 145 \%$ & $8.8 \% / 20.4 \%$ \\
\hline Test IV & ID9-12 & $8.8 \% / 178 \%$ & $8.5 \% / 200 \%$ & $5.3 \% / 21 \%$ \\
\hline Test V & ID3-5,7-12 & $4.8 \% / 199 \%$ & $5.4 \% / 320 \%$ & $9 \% / 70 \%$ \\
\hline Test VI & ID3-5,7-8,12 & $5.1 \% / 153 \%$ & $5.4 \% / 123 \%$ & $9.3 \% / 30 \%$ \\
\hline
\end{tabular}

Source: Authors

The g\&h distribution proved to be a very suitable for operational risk modelling. Its results were consistent, as the extreme worst case and the average loss custom events were being added to the data sample - this conclusion corresponds with the findings of Degen, Embrechts, Lambrigger (2007). The parameter estimates differ based on the number of the additional extreme events used for the scenario analysis; as the more extreme losses were added to the data sample the higher the estimate for $\hat{h}$ and $\hat{g}$ was and so the higher were the losses generated during the loss aggregation procedure.

The $g \& h$ distribution is, unlike the EVT, consistent even if less extreme but more frequent average loss cases are added to the data sample. In the average loss case the custom losses were of very similar magnitude as the most severe empirical losses. Even if all the scenarios were considered, the estimated regulatory capital would not exceed $12 \%$ of the Basel Income Ratio suggesting that BANK would be able to handle the losses of such high magnitude.

\subsection{Implications for the financial institution}

As mentioned above, the scenario analysis added the custom hypothetical losses to the original loss database. Since all those events impose extreme losses, it was assumed that the estimates of the regulatory capital charge as well as of the economic capital would significantly increase.

In the cases where extreme worst-case losses were considered the final estimates for regulatory capital charge spiked up to $90 \%$ of the Basel Income Ratio ${ }^{5}$. Such huge amount of capital cannot be set aside to cover risks, because it would make the financial institution noncompetitive - the cost of its capital would be much higher than the industry average. On the other hand, it is hardly reasonable to expect that all the worst case scenarios will ever happen concurrently in such short time period that was considered throughout this paper -4 years. But even if a longer time period - like 10 or 20 years - would be considered, the probability that the worst case joint scenario from Test I would occur is close to zero.

5 This means 6 times higher capital requirement than in case of BIA. 
From this point of view it seems more reasonable to work with average loss joint scenario cases, which have a higher probability of occurrence - in some cases over $2 \%$. The tests that employed the average losses provided a higher but still realistic level of capital estimates - up to $12 \%$ of the Basel Income Ratio for the capital charge.

It was shown that the combination of the scenario analysis and the LDA approach can improve applicability and soundness of the capital estimates over the methods, where just historical data are used. Since new internal and external operational risk data will be added to the loss databases in the future, the quantitative LDA techniques will be more important. Even though, it would be still valuable to consider plausible events and evaluate, what would be the impact of these events. After all of the tests were run we can say that BANK would be able to survive losses imposed by the average joint scenario combination. The losses defined in the worst-case scenarios are so extreme, that the bank would have to take the risks in order not to increase the cost of capital to an unacceptable level.

As BANK can be considered a typical bank in the CEE region, it can be concluded that similar results would apply to other banks. However, it is up to future research to prove or reject this hypothesis.

\section{Conclusion}

The main aim of this paper was to evaluate the appropriateness of capital estimates based on historical loss events and to measure the impact of plausible operational risk events that were added to the empirical loss data sample provided by an anonymous Central European bank. The technique presented in this paper claims to be consistent and applicable for other financial institutions. There were two main questions the paper was aimed to answer:

- What is the appropriate statistical method to model the operational risk loss data distribution and to measure reasonable capital estimates for the institution?

- What is the impact of extreme events defined in extreme case scenarios on the capital estimates and on the financial institution?

The evaluation of the operational risk exposure measurement employed different statistical methods and distributions - the most important ones were the EVT and the g\&h distribution. For the original data sample the results for the EVT seemed consistent, statistically significant and economically reasonable. However, after the custom extreme events were added to the data sample, both EVT methods started to provide very inconsistent estimates. So the EVT method does not seem suitable to model the operational risk data even if it is widely favored by many researchers such as Degen, Embrechts, Lambrigger (2007) or Chernobai, Rachev and Fabozzi (2007).

The alternative method to the EVT was the g\&h distribution, which was evaluated as the most suitable from all the parametric distributions used, what confirms findings of Degen, Embrechts, Lambrigger (2006) or Dutta, Perry (2007). It proved itself very resilient to contamination and outlier observations and it provided very reasonable results even while very extreme worst-case losses were considered. 
So the answer to the first question would be that the most suitable method to model the operational risk loss data distribution is to use the $\mathrm{g} \& \mathrm{~h}$ distribution which is able to model the whole data sample without trimming or truncating the data in an arbitrary or subjective manner as suggested by Dutta, Perry (2007). There might be other statistical distributions that are able to measure and model the tail structure of the operational risk data - we believe that a further research will be devoted to this question and even more suitable measurement methods will be developed.

The answer to the second question is that, given the reasonable definition of the scenario analysis and the loss amounts defined under scenarios, the estimated regulatory capital charge has increased significantly but still to a level which is acceptable for the financial institution. The operational risk assessment method should be reasonable for the regulator as well and so this paper provides a framework of how to combine the scenario analysis with the LDA approach. Using the scenario analysis can also help the financial institution to mitigate the operational risk and to decrease the impact of potential losses. This framework can be used for future application and the impact of other scenarios can be assessed.

Some further questions and tasks remain open, however. The external data could be merged with internal data in order to better capture the potential impact of events that have not happened to the financial institution yet. Statistical differences between the business lines and the event types should be analyzed. Robust methods or alpha stable distributions can be used as suggested by Chernobai, Rachev and Fabozzi (2007). Other EVT methods, particularly for the threshold estimation, could be used. Also it should be further explored whether the characteristic of operational risk exposure is somewhat similar among bank in one region. However, this issue goes beyond the scope of this paper and is left for future consideration.

\section{References}

Arai, T. (2006), "Key Points of Scenario Analysis." Bank of Japan, 2006, http://www.boj.or.jp/en/type/ release/zuijinew/data/fsc0608be2.pdf

BCBS (2006), "International Convergence of Capital Measurement and Capital Standards." (A Revised Framework Comprehensive Version.) Basel Committee on Banking Supervision, Bank for International Settlement, Basel June 2006, http://www.bis.org/publ/bcbs128.pdf.

Chalupka, R., Teplý, P. (2008), "Operational Risk Management and Implications for Bank's Economic Capital - A Case Study." IES Working Papers 17/2008, http://ies.fsv.cuni.cz/sci/publication/show/ id/3477/lang/cs.

Chernobai, A., Rachev, S., Fabozzi, F. (2007), Operational Risk. A Guide to Basel II Capital Requirements, Models and Analysis. John Willey \& Sons, Inc., March 2007. ISBN: 0470148780.

Cihak, M. (2004), "Designing Stress Tests for the Czech Banking System." CNB Internal Research and Policy Note 03/2004, http://www.cnb.cz/en/research/research_publications/irpn/download/irpn_3_2004.pdf

Dutta, K., Perry, J. (2007), "A Tale of Tails: An Empirical Analysis of Loss Distribution Models for Estimating Operational Risk Capital" Working Paper 06-13, Federal Reserve Bank of Boston, Boston January 2007, http://www.bos.frb.org/economic/wp/wp2006/wp0613.pdf

de Fontnouvelle, P., Jordan, J., Rosengren, E. (2005), "Implications of Alternative Operation Risk Modeling Techniques." NBER Working Paper Series, Cambridge February 2005, http://www.nber. org/papers/w11103.pdf

de Fontnouvelle, P., de Jesus-Rueff, V., Jordan, J., Rosengren, E. (2003), "Using Loss Data to Quantify Operational Risk." Technical Report, Federal Reserve Bank of Boston and Fitch Risk. 
Degen, M., Embrechts, P., Lambrigger, D. (2007), "The Quantitative Modelling of Operational Risk: Between g-and-h and EVT." ETH Zurich 2007, http://www.math.ethz.ch/ degen/g-and-h.pdf

Embrechts, P., Frey, R., McNeil, A. (2005), "Quantitative Risk Management: Concepts, Techniques and Tools." Princeton Series in Finance.

Jorion, P. (2007), Value at Risk: The New Benchmark for Managing Financial Risk. $3^{\text {rd }}$ edition, McGrawHill 2007.

Kuhn, R., Neu, P. (2004), "Adequate Capital and Stress Testing for Operational Risks." Dresdner Bank AG 2004, http://www.gloriamundi.org/picsresources/rkpn2.pdf.

Mejstřík, M., Pečená, M., Teplý, P. (2008), Basic Principles of Banking. Prague: Karolinum Press.

Mignola, G., Ugoccioni, R. (2007), "Statistical Approach to Operational Risk Management." Sampolo IMI Group Italy 2007, http://www.r-project.org/user-2006/Abstracts/Mignola+Ugoccioni.pdf.

Rippel, M. (2008), "Operational Risk - Scenario Analysis.” IES FSV UK Diploma Thesis 2008.

Rippel, M., Teplý, P. (2010), Stress Testing and Scenario Analysis. Saarbrücken: VDM Verlag.

Teplý, P. (2010), The Truth about the 2008-2009 Crisis: A Hard Lesson for The Global Markets. Saarbrücken: VDM Verlag.

Rosengren, E. (2006), "Scenario Analysis and the AMA." Federal Reserve Bank of Boston, 2006, http://www.bos.frb.org/bankinfo/qau/presentations/2006/er71906.pdf. 